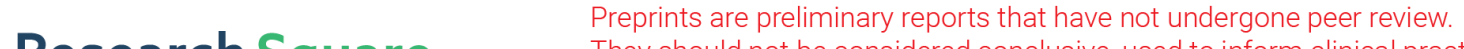 They should not be considered conclusive, used to inform clinical practice, or referenced by the media as validated information. \\ Expression of TCF7L2 in Glioma and Relation With Clinicopathological Characteristics and Patient Overall Survival
}

\section{S.-Y. JING}

Capital Medical University Sanbo Brain Hospital

\section{CHEN}

Capital Medical University Sanbo Brain Hospital

\section{S. HAN}

Capital Medical University Sanbo Brain Hospital

N. LIU

Capital Medical University Sanbo Brain Hospital

\section{M.-Y. HAN}

Capital Medical University Sanbo Brain Hospital

\section{Y.-K. YANG}

Capital Medical University Sanbo Brain Hospital

Chang-xiang Yan ( $\sim$ peter6120636@163.com )

Capital Medical University Sanbo Brain Hospital

\section{Research article}

Keywords: Glioma, TCF7L2, Overall Survival, Prognosis, clinicopathological characteristics

Posted Date: December 21st, 2020

DOl: https://doi.org/10.21203/rs.3.rs-130738/v1

License: (c) (i) This work is licensed under a Creative Commons Attribution 4.0 International License. Read Full License 


\section{Abstract}

Background: TCF7L2 gene is known as transcription factor 7-like 2 which has been identified as a novel transcription factor epithelial-mesenchymal transition (EMT) in tumor cells at 10q25.3. TCF7L2 may affect cancer progression and plays a central role in cancer proliferation, migration and invasion. However, its clinical and prognostic value have not been researched in glioma. The purpose of our study was to research TCF7L2 expression and evaluate the clinical value of prognosis.

Method: We collected glioma specimens including low-grade glioma $(n=46)$ and glioblastoma $(n=51)$ from September 2015 to September 2017.Expression of TCF7L2 in 97 specimens were detected by quantitative real-time PCR (qRT-PCR).The chi-square test was applied to analyze the relationship between TCF7L2 expression and clinicopathological characteristics. The overall survival (OS) was analyzed by binary logistic regression analysis, the survival curves were drew by Kaplan-Meier. Univariate and multivariate analysis were utilized to analyze the relationship between prognosis and clinicopathological characteristics including TCF7L2 expression.

RESULTS: Compared with low-grade glioma group, the expression of TCF7L2 was significantly increased $(p<0.05)$. TCF7L2 overexpression was associated with large tumor volume $(p=0.03)$, higher WHO grade $(p=0.001)$, and recurrence $(p=0.001)$. Moreover, Kaplan-Meier analysis proved that overexpressed TCF7L2 was related with poor OS ( $p<0.05)$. The multivariate analysis suggested that TCF7L2 expression was an independent prognostic factor.

CONCLUSIONS: Our research proved that TCF7L2 was over- expressed in glioblastoma, and, related with tumor prognosis, which, therefore, could be an independent prognostic factor for glioma patients.

\section{Background}

Glioblastoma is the common malignant brain tumor owing to its stronger invasive ability and resistance to treatment. The morbidity rate of glioblastoma is approximate $0.59-0.69 / 100000$ people worldwide, affecting those aged 20 years or older, especially those aged 40 to 70 years, with a median age of onset of 63 years ${ }^{1}$. The morbidity is higher in male $(3.97 / 100000)$ than in females ${ }^{2,3}(2.53 / 100000)$.

According to NCCN Guidelines, the standard therapies include surgery, radiotherapy with concomitant temozolomide (TMZ), adjuvant TMZ chemotherapy and TTF therapies,but the 5 -year OS is only $9.8 \%{ }^{3}$. Although with comprehensive therapy,the median survival time is approximately 12-15 months for GBM patients after diagnosis ${ }^{4,5}$. Therefore, it is necessary to understand the mechanism of GBM and discuss its potential as a therapeutic target for GBM patients.

The Wnt/ $\beta$-catenin signaling pathway is important in majority tumor progression.TCF7L2 is a member of the $W n t / \beta$-catenin signaling pathway,play an important role in metabolism, cell differentiation/ proliferation and cell death ${ }^{6}$.We collected the samples including $51 \mathrm{GBM}$ tissues and 46 low-grade glioma tissues $\mathrm{WHO}(\mathrm{I}-\mathrm{II})$.We focused on the TCF7L2 effect and the expression level in glioma tissue. 
Subsequently,we analyzed the relation between TCF7L2 expression level and clinicopathological characteristics, and investigated the prognosis factors.

\section{Method}

\section{Patients and Tissue Samples}

Patients who were performed initial surgery in The Sanbo Brain Hospital of Capital Medical University from September 2015 to September 2017,were retrospectively selected for this research. All patients did not receive chemotherapy or radiotherapy prior to surgery. All samples were diagnosed by pathologists and stored in liquid nitrogen .

The patients' characteristics are described in Table I. Tissue samples usage was approved by Ethics Committee of the Sanbo Brain Hospital of Capital Medical University and written informed consent was obtained from all study participants.

\section{RNA Extraction and qRT-PCR Analyses}

The total RNA was extracted from the sample by using TRIzol reagent (Invitrogen, Carlsbad, CA, USA).RNA was reverse transcribed to complementary DNA (cDNA) by using a PrimeScript RT reagent kit (Thermo, Beijing, China). Its synthesis was conducted at $37^{\circ} \mathrm{C}$ for $15 \mathrm{~min}$, then $85^{\circ} \mathrm{C}$ for $5 \mathrm{~s}$ according to the experimental protocols. Real-time PCR reactions were carried by Applied Biosystems 7500. Real-time PCRs was carried in triplicate.The relative TCF7L2 expression was computed and normalized using the 2$\triangle \mathrm{ct}$ method relative to GAPDH.The primers for TCF7L2:5'-TGCTCTGCGGTTGCTATGTTGAC-3',3'GCTGCGAGT CCTCACCAATGTC-5'and for GAPDH:5'-CAGACCACAGTCCATGC CATCAC-3'®3'GACGCCTGCTTCACCACCTTC-5'

\section{Data Analysis}

The comparison of the TCF7L2 expression level between GBM and low-grade glioma was performed by the two-sample Student's t-test. The chi-square test were used to examine the associations between TCF7L2 expression and the clinicopathological characteristics.

In addition, survival curves were drew by the Kaplan-Meier method and analyzed with log-rank test. Cox proportional-hazards regression analysis was applied to estimate univariate and multivariate hazard ratios for OS. A value of $\mathrm{P}<0.05$ was considered as significantly different. SPSS software 20.0 was applied in the present study.

\section{Results}

\section{TCF7L2 was Upregulated in Glioblastoma Tissues}


We measure TCF7L2 expression levels in 97 patients with glioma by the qRT-PCR. The data revealed that TCF7L2 had higher expression in GBM tissues than the low-grade group ( $<<0.05$, Fig. 1).

\section{Relation Between TCF7L2 Expression and Clinicopathological Factors of GBM Patients}

We found the TCF7L2 high expression in GBM.The median expression level of TCF7L2 was used as a dividing point.we divide 97 patients into two groups (high and low expression). Table I summarizes the relation between TCF7L2 expression and clinicopathological parameters in glioma.The results showed that TCF7L2 high expression was significantly related with larger tumor size $(p=0.03)$, higher WHO grade $(p=0.001)$ and recurrence $(p=0.001)$.

\section{Upregulation of TCF7L2 Confers Poor Prognosis in Patients}

Univariate and multivariate analysis were utilized to evaluate the association between OS and various clinicpathological features including TCF7L2 expression level (Table I).

Kaplan-Meier method indicated that the five-year OS of patients was significantly shorter in patients with high TCF7L2 expression than in those with low TCF7L2 expression $(p<0.05)$,but there were no significantly differences in two-year OS $(p>0.05)$. The OS was significantly longer with small tumor size( $\leqq 3 \mathrm{~cm})$ compared to those with large tumor size $(>3 \mathrm{~cm})(\mathrm{p}<0.05)$. The OS was significantly shorter in patients with recurrence than those with no recurrence $(p<0.05)$, but was significantly longer in patients with low grade group compared to those with glioblastoma $(p<0.05)$. There were no significantly differences between necrosis and non-necrosis patients ( $p>0.05)$ (Fig. 2,3).

Multivariate analysis confirmed that TCF7L2 expression,WHO grade and recurrence was the independent prognostic factors for patients with glioma in five-year OS. Their hazard ratios were $4.91(95 \% \mathrm{Cl}, 2.49-$ 9.69),55.6 (95\%Cl,14.9-207.4),3.00(95\%Cl,1.31-6.86), respectively ( $<$ < 0.05) (Table II, Fig. 4).

Table I. Relation of the expression level of TCF7L2 with clinicopathological parameters 


\begin{tabular}{|c|c|c|c|c|c|}
\hline \multirow[t]{2}{*}{ Characteristic } & \multirow[t]{2}{*}{ Case number } & \multicolumn{2}{|c|}{ TCF7L2 expression } & \multirow[t]{2}{*}{$\mathbf{R}_{5}$} & \multirow[t]{2}{*}{$p$-valu } \\
\hline & & $\operatorname{Low}(n=47)$ & $\mathrm{High}(\mathrm{r}$ & & \\
\hline Age(years) & & & & & 0.98 \\
\hline$\leq 45$ & 35 & 17 & 18 & & \\
\hline$>45$ & 62 & 30 & 32 & & \\
\hline Gender & & & & & 0.97 \\
\hline Male & 60 & 29 & 31 & & \\
\hline Female & 37 & 18 & 19 & & \\
\hline Tumor size $(\mathrm{cm})$ & & & & 0.21 & 0.03 \\
\hline$\leq 3$ & 37 & 20 & 13 & & \\
\hline$>3$ & 60 & 23 & 37 & & \\
\hline Necrosis & & & & & 0.18 \\
\hline Yes & 47 & 24 & 23 & & \\
\hline No & 50 & 23 & 27 & & \\
\hline WHO grade & & & & 0.44 & 0.001 \\
\hline I-II & 46 & 33 & 13 & & \\
\hline IV & 51 & 14 & 37 & & \\
\hline Recurrence & & & & 0.28 & 0.001 \\
\hline Yes & 57 & 21 & 36 & & \\
\hline No & 40 & 26 & 14 & & \\
\hline
\end{tabular}

Table II. Univariate and multivariate analysis of 5-year OS. 


\begin{tabular}{|lllllll|}
\hline Parameter & \multicolumn{2}{l}{ Univariate } & \multicolumn{5}{c|}{ Multivariate } \\
\hline & HR & $95 \%$ Cl & P & HR & $95 \%$ Cl & P \\
\hline Age & 1.79 & $10.71-4.50$ & 0.21 & & & \\
$(\leq 45 />45)$ & & & & & & \\
\hline $\begin{array}{l}\text { Sex } \\
\text { (male/femal) }\end{array}$ & 0.92 & $0.37-2.31$ & 0.87 & & & \\
\hline $\begin{array}{l}\text { Tumor size } \\
\text { ( } \leq 3 \text { cm/>3 cm) }\end{array}$ & 1.77 & $1.07-2.92$ & 0.02 & & & \\
\hline $\begin{array}{l}\text { Necrosis } \\
\text { (yes/no) }\end{array}$ & 1.68 & $0.67-4.19$ & 0.26 & & & \\
\hline $\begin{array}{l}\text { Recurrence } \\
\text { (yes/no) }\end{array}$ & 6.85 & $3.53-13.2$ & 0.001 & 3.00 & $1.31-6.86$ & 0.001 \\
\hline $\begin{array}{l}\text { WHO grade } \\
\text { (I-II/IV) }\end{array}$ & 38.6 & $12.8-116.2$ & 0.001 & 55.6 & $14.9-207.4$ & 0.001 \\
\hline $\begin{array}{l}\text { TCF7L2 expression } \\
\text { (high/low) }\end{array}$ & 1.83 & $1.12-2.98$ & 0.01 & 4.91 & $2.49-9.69$ & 0.001 \\
\hline
\end{tabular}

\section{Discussion}

TCF7L2 has been reported to have an effect on the surpression of cell proliferation, migration and invasion in the literature ${ }^{7}$. The transcription factor 7-like 2 (TCF7L2) gene may affect cancer development and prognosis because the TCF7L2 gene play an important role in the $\mathbf{W n t} / \boldsymbol{\beta}$-catenin signaling pathway ${ }^{8,9}$.

To date, various biological markers have been reported in glioma ${ }^{10,11}$. TCF7L2, represents a central factor in metabolism, cell proliferation, and cell apoptosis ${ }^{12,13}$. Bo Yu ${ }^{7}$ et al.found that TCF7L2 overexpression increased cell viability,migration and invasion in the cells with CRNDE inhibition. The TCF7L2 expression and prognostic value in glioma has not yet been reported.

In our study, we detected significantly higher TCF7L2 expression in GBM tissues than in low-grade group. Moreover,TCF7L2 overexpression was significantly related with larger tumor size, higher WHO grade and necrosis.

Our study reported shorter OS in patients with higher expression, however, there was no significant difference in 2-year OS in the two groups. We found that the 2-year OS was relatively high in the patients 
who underwent standard therapies including radiotherapy with concomitant temozolomide (TMZ), and adjuvant TMZ after surgery,in the high expression group. There was statistically significance in 5-year survival rate $(p<0.05)$.

Multivariate analysis revealed that WHO grade, tumor recurrence and TCF7L2 expression level were independent prognositic factors for OS. The hazard ratio in high TCF7L2 expression group was 4.91 times more than low expression group $(\mathrm{p}<0.05,95 \% \mathrm{Cl}, 2.49-9.69)$.TCF7L2 expression was independently related with the OS, indicating that higher TCF7L2 level was a marker of poor prognosis for patients.

\section{Conclusions}

TCF7L2 could be a potential prognostic factor and therapeutic target for patients with glioma.The underlying molecular mechanisms of TCF7L2 involvement in the Wnt/ $\beta$-catenin signaling pathway needs to be investigated in future studies.

\section{Abbreviations}

TCF7L2 Transcription factor 7-like 2

qRT-PCR Real-time quantitative PCR

OS Overall survival

TMZ Temozolomide

EMT Epithelial-mesenchymal transition

TTF Tumor Treating Fields

GBM Gliomablastoma

cDNA Complementary DNA

HR Hazard ratios

$\mathrm{Cl}$ Confidence interval

\section{Declarations}

\section{Ethics approval and consent to participate}

The experimental protocol was established, according to the ethical guidelines of the Helsinki Declaration and was approved by the Human Ethics Committee of Capital Medical University Sanbo Brain Hospital. Written informed consent was obtained from individual or guardian participants. 


\section{Consent to publish}

Not applicable.

\section{Availability of data and materials}

All data generated or analysed during this study are included in this published article.

\section{Competing interests}

The authors declare that they have no competing interests

\section{Funding}

Not applicable

\section{Authors' Contributions}

L. CHEN, S. HAN and N. LIU analyzed and interpreted the glioma patient data. S.-Y. JING performed the qRT-PCR of the glioma tissues, C.-X. YAN and S.-Y. JING were major contributors in writing the manuscript. All authors read and approved the final manuscript.

\section{Acknowledgements}

We appreciated W.-Y DENG for providing constructive suggestions to the manuscript.

\section{References}

1. Darlix A, Zouaoui S, Rigau V, Bessaoud F, Figarella Branger D, MathieuDaudé H, Trétarre B, Bauchet F, Duffau H,Taillandier L,Bauchet L.Epidemiology for primary brain tumors: a nationwide populationbased study. J Neurooncol.2017; 131: 525-546.

2. Ostrom QT,Gittleman H,Fulop J,Liu M,Blanda R,Kromer C,Wolinsky Y,Kruchko C, Barnholtz-Sloan JS.CBTRUS statistical report: primary brain and central nervous system tumors diagnosed in the United States in 2008-2012.Neuro Oncol.2015; 17: iv1-iv62.

3. Koshy M,Villano JL,Dolecek TA,Howard A,Mahmood U,Chmura SJ, Weichselbaum RR,McCarthy BJ.Improved survival time trends for glioblastoma using the SEER 17 population-based registries. J Neuro-Oncol.2012; 107(1): 207-212.

4. Stupp R,Hegi ME, Mason WP,Van den Bent MJ, Taphoorn MJB, Janzer RC, Ludwin SK,Allgeier A,Fisher B,Belanger K,Hau P,Brandes AA,Gijtenbeek J, Marosi C,Vecht CJ,Mokhtari K,Wesseling P,Villa S,Eisenhauer E,Gorlia T,Weller M, Lacombe D,Cairncross JG,Mirimanoff RO. Effects of radiotherapy with concomitant and adjuvant temozolomide versus radiotherapy alone on survival in glioblastoma in a randomised phase III study: 5-year analysis of the EORTC-NCIC trial.Lancet Oncol.2009; 10: 459466. 
5. Stupp R, Mason WP, van den Bent MJ, Weller M, Fisher B, Taphoorn MJB, Belanger K,Brandes AA,Marosi C,Bogdahn U,Curschmann J,Janzer RC,Ludwin SK,Gorlia T,Allgeier A,Lacombe D,Cairncross JG,Eisenhauer E,Mirimanoff RO. Radiotherapy plus concomitant and adjuvant Temozolomide for glioblastoma. N Engl J Med.2005; 352: 987-996.

6. Hrckulak D, Kolar M, Strnad H, Korinek V.TCF/LEF Transcription Factors: An Update from the Internet Resources.Cancers (Basel) 2016; 8(7): 70.

7. Yu B,Ye X,Du Q,Zhu B, Zhai Q, Li XX.The Long Non-Coding RNA CRNDE Promotes Colorectal Carcinoma Progression by Competitively Binding miR-217 with TCF7L2 and Enhancing the Wnt/ $\beta$ Catenin Signaling Pathway.Cell Physiol Biochem 2017; 41: 2489-2502.

8. Stefani Spranger, Riyue Bao, Thomas F Gajewski.Melanoma-intrinsic $\beta$-catenin signalling prevents anti-tumour immunity.Nature 2015; 523: 231-235.

9. Zhao C, Deng Y, Liu L, Yu K, Zhang L, Wang H, He X, Wang J, Lu C, Wu LN, Weng Q, Mao M, Li J, van Es JH, Xin M, Parry L, Goldman SA, Clevers H, Lu QR.Dual regulatory switch through interactions of TCF712/TCF4 with stage-specific partners propels oligodendroglial maturation. Nat Commun 2016; 7: 10883.

10. QU DW, XU HS, HAN XJ, WANG YL, OUYANG CJ. Expression of cyclinD1 and Ki-67 proteins in gliomas and its clinical significance.Eur Rev Med Pharmacol Sci 2014; 18: 516-519.

11. LI W, XIE P, RUAN WH. Overexpression of LnCRNA UCA1 promotes osteo- sarcoma progression and correlates with poor prognosis. J Bone Oncol 2016; 5: 80- 85.

12. Chen C, Cao FQ, Bai LP,Liu Y, Xie JL,Wang W,Si Q,Yang J, Chang AT, Liu D, Liu DC,Chuang TH, Xiang R,Luo YP.IKK $\beta$ Enforces a LIN28B/TCF7L2 Positive Feedback Loop That Promotes Cancer Cell Stemness and Metastasis. Cancer Res 2015; 75(8): 1725-1735.

13. Weng QJ, Tan BQ,Wang JJ,Wang J,Zhou H,Shi J,He QJ,Yang B.5-Fluorouracil causes severe CNS demyelination by disruption of TCF7L2/HDAC1/HDAC2 complex in adolescent mice.Toxicology 2014; 325: 144-150.

\section{Figures}




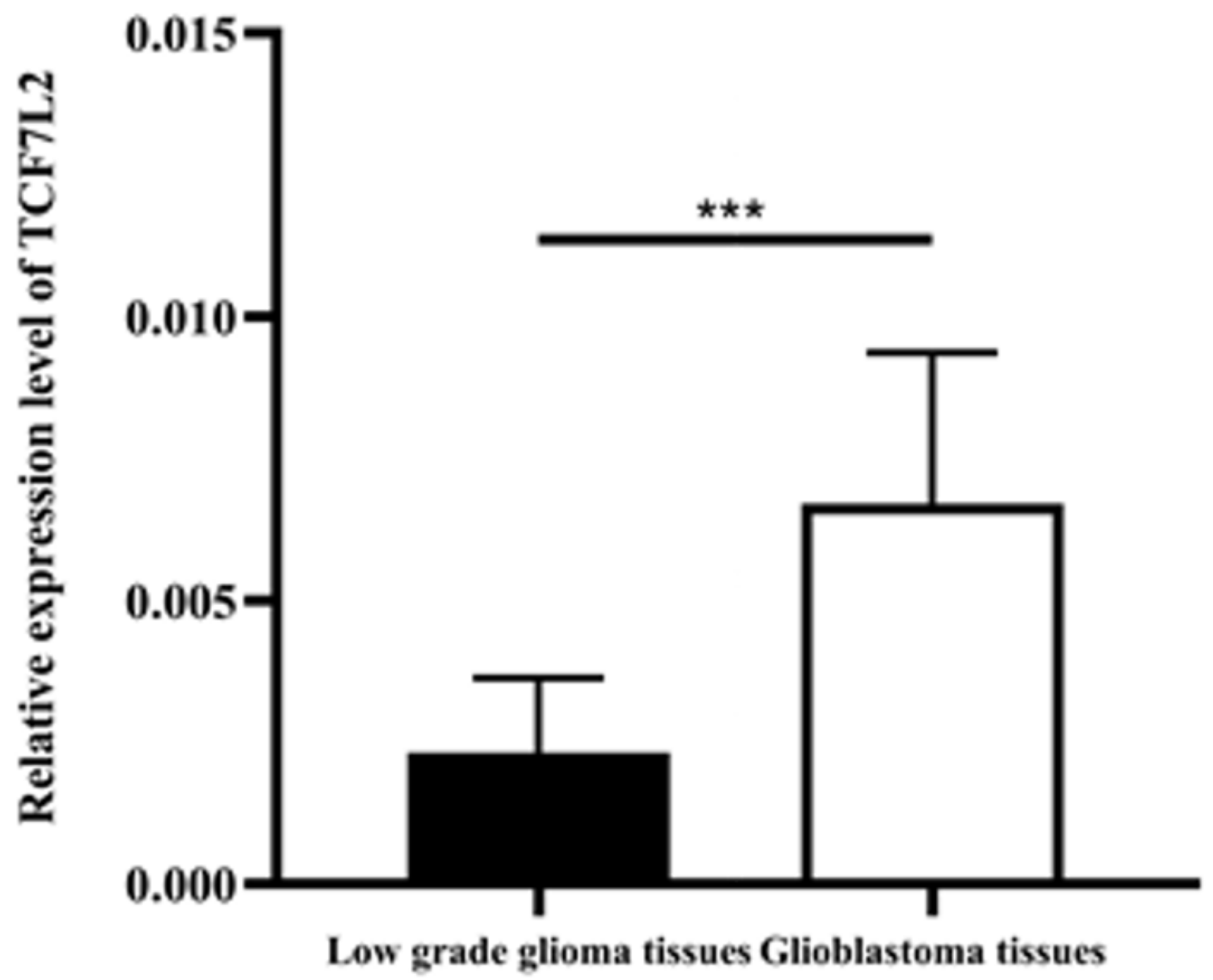

Figure 1

Expression levels of TCF7L2 in GBM tissues were significantly higher $(* \star \star p<0.05)$. 
A

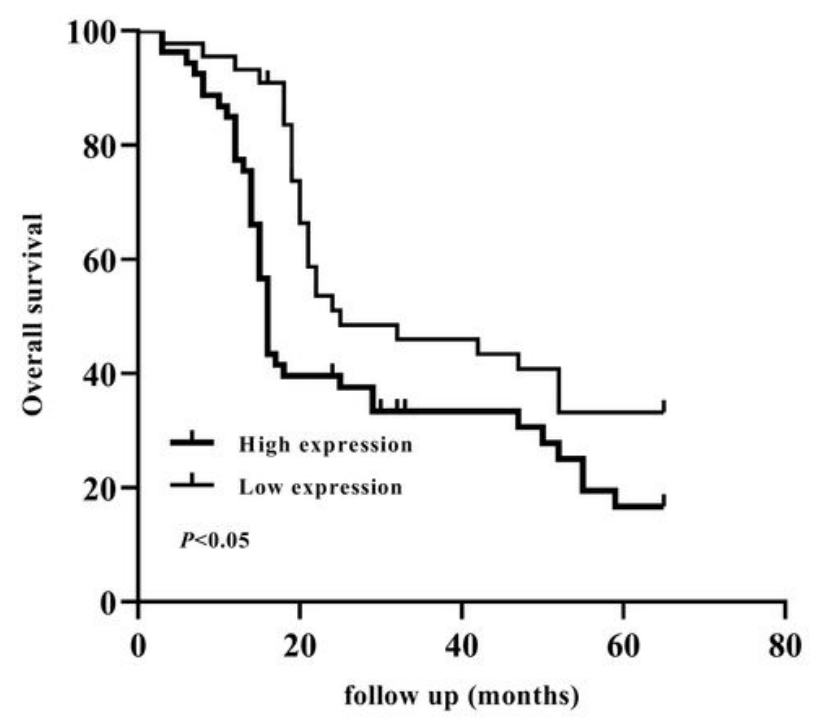

C

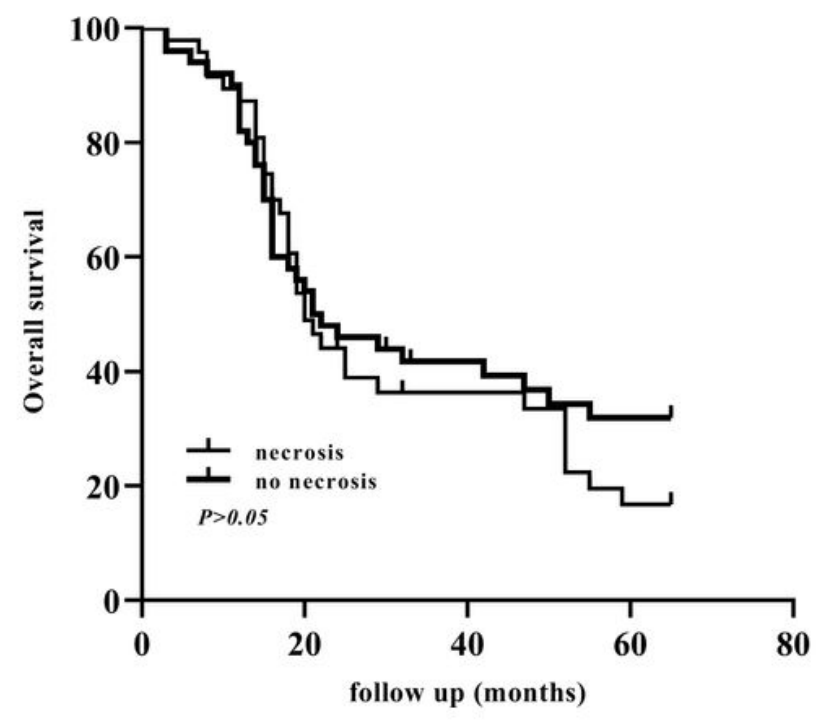

B

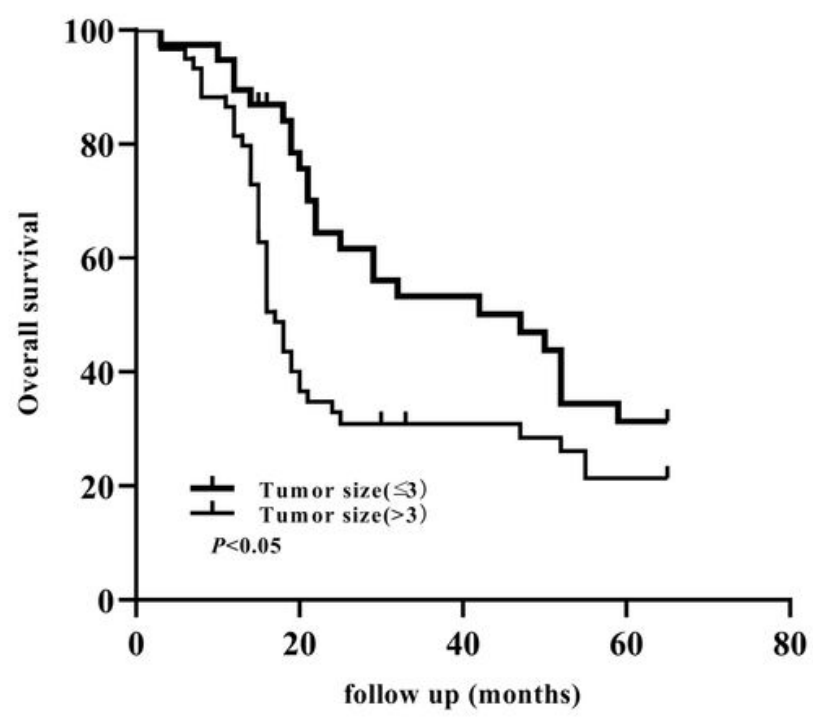

D

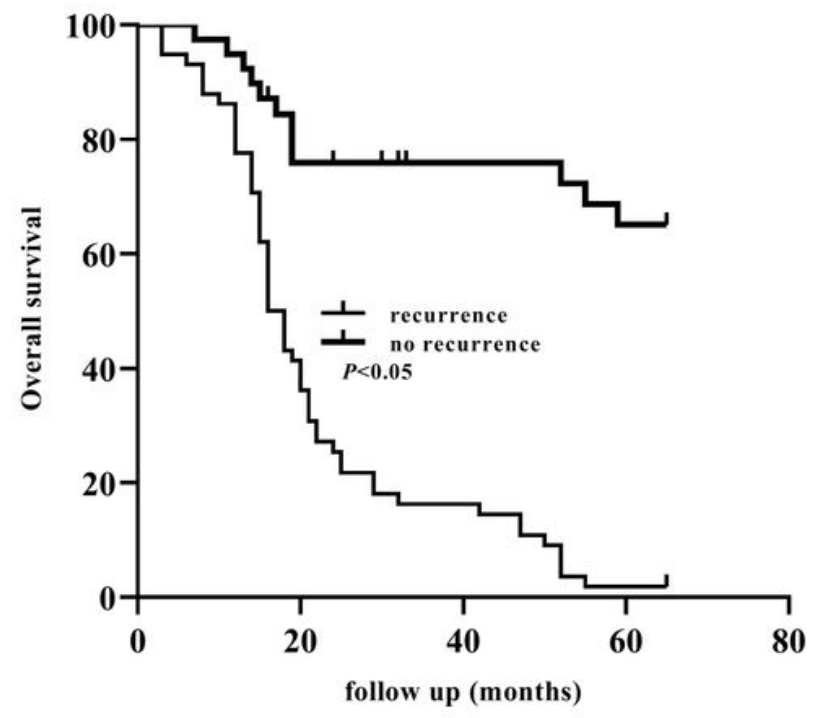

Figure 2

Kaplan-Meier 5-year OS curves of patients with glioma according to clinicopathological features. A: TCF7L2 higher expression group showed shorter OS compared with patients with lower expression $(p<0.05), B$ : Patients with larger tumor size $(>3 \mathrm{~cm})$ showed worse OS compared with patients with small tumor size $(\leqq 3 \mathrm{~cm})(\mathrm{p}<0.05), C$ :Patients with necrosis showed no significantly differences compared with those with non-necrosis $(p>0.05), D: P a t i e n t s$ with recurrence indicated worse OS compared with those with no recurrence $(p<0.05)$. 
A

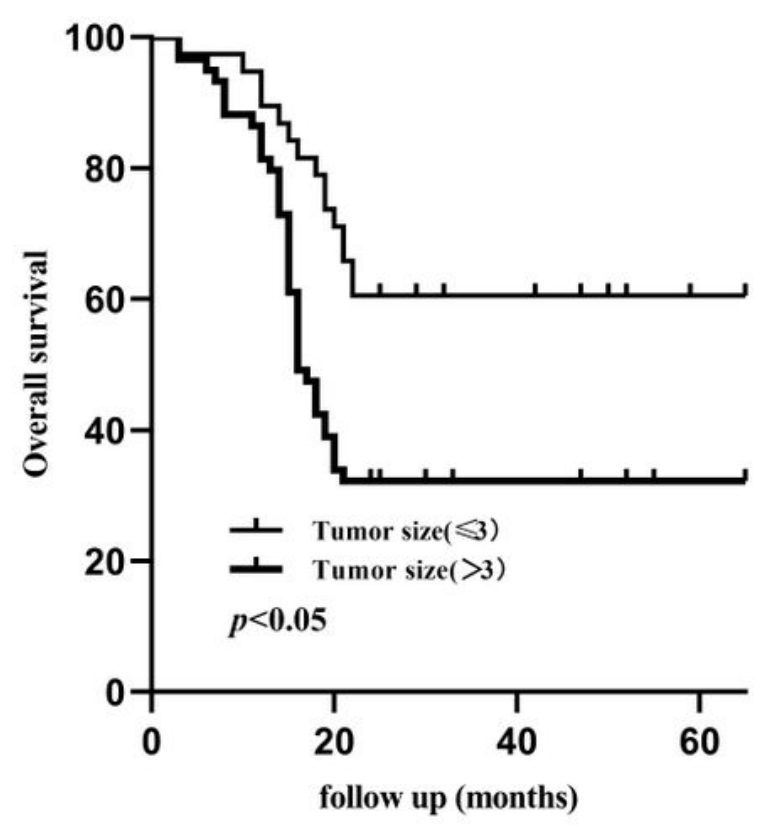

C

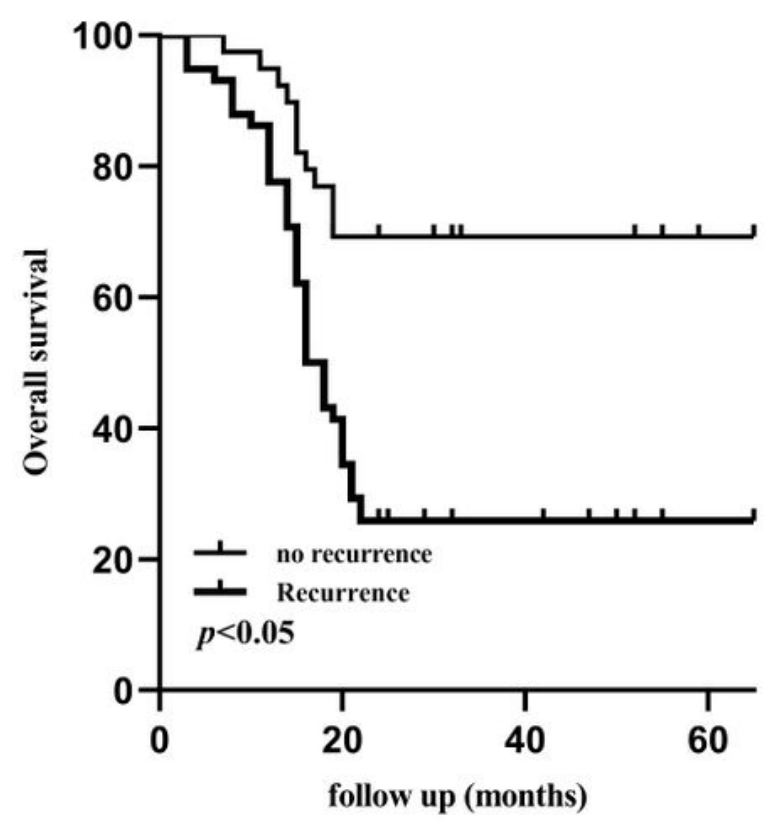

B

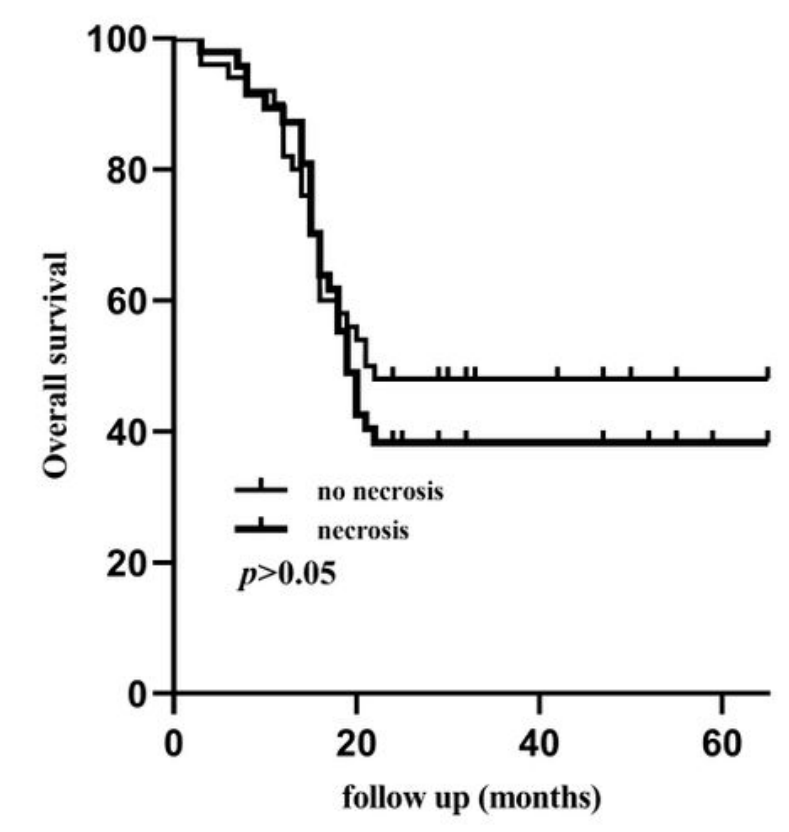

D

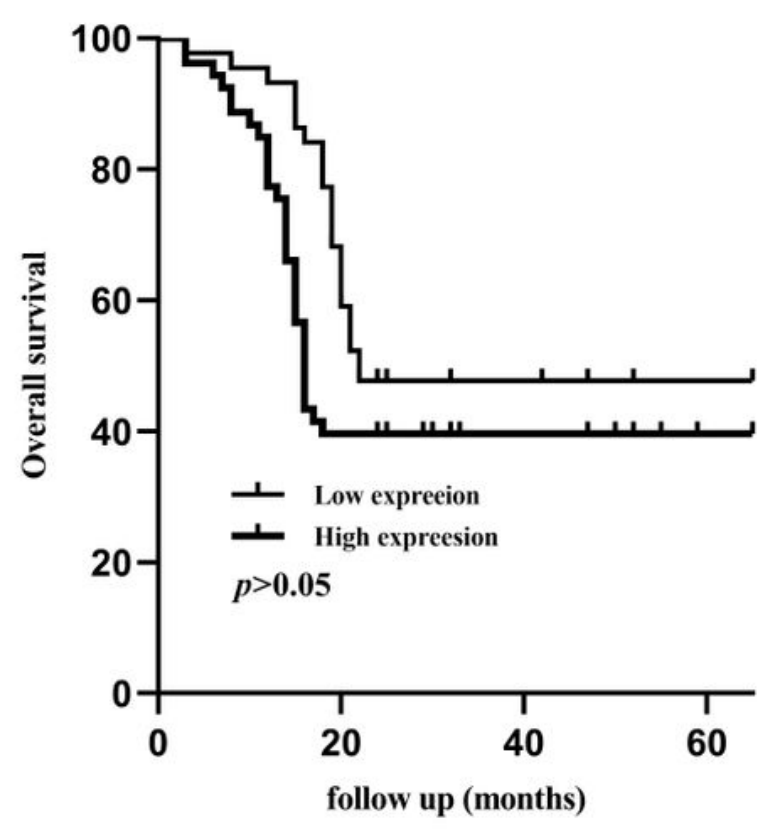

\section{Figure 3}

2 year OS curves of patients with glioma according to clinicopathological features A:Larger tumor size group $(>3 \mathrm{~cm})$ showed poor OS compared with small tumor size group $(\leqq 3 \mathrm{~cm})(p<0.05)$, B:Patients with necrosis showed no significantly differences compared with those with no necrosis $(p>0.05), C$ : Patients with recurrence indicated worse OS compared with those with no recurrence $(p<0.05), D$ :Patients with 
higher expression of TCF7L2 showed no significantly differences compared with those with lower expression $(p>0.05)$.

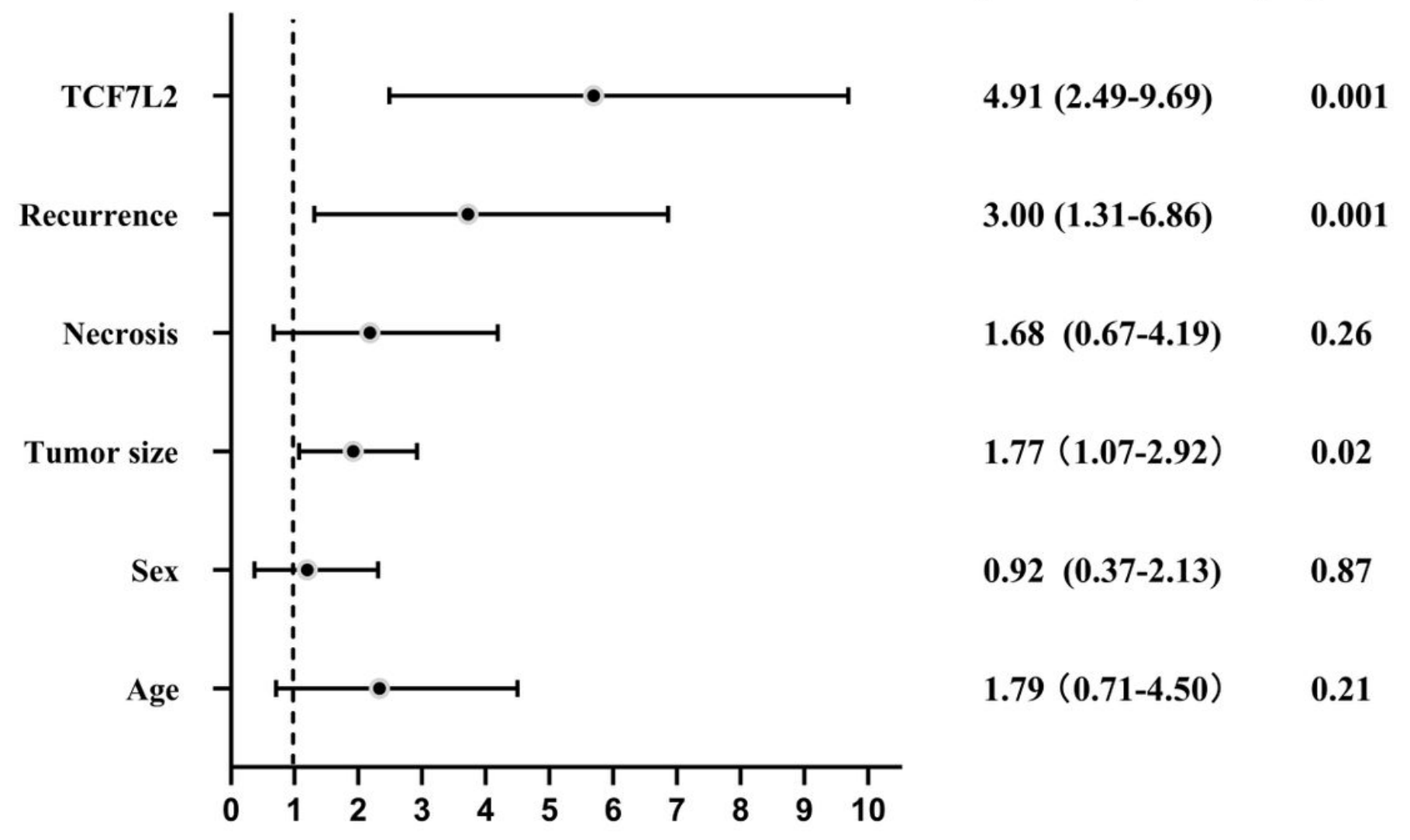

Figure 4

Forest plots analysis with 5-year OS with 95\% confidence interval for clinicopathological features. 